\title{
River classification and change detection from landsat images by using a river classification toolbox
}

\author{
Supattra Puttinaovarat ${ }^{1}$, Aekarat Saeliw ${ }^{2}$, Siwipa Pruitikanee ${ }^{3}$, Jinda Kongcharoen ${ }^{4}$, \\ Supaporn Chai-Arayalert ${ }^{5}$, Kanit Khaimook ${ }^{6}$, Paramate Horkaew ${ }^{7}$ \\ $1,2,3,4,5$ Faculty of Science and Industrial Technology, Prince of Songkla University, Surat Thani Campus, Surat Thani, \\ Thailand \\ ${ }^{6}$ Ramkhamhaeng University, Bangkok, Thailand \\ ${ }^{7}$ School of Computer Engineering, Institute of Engineering, Suranaree University of Technology, Nakhon Ratchasima,
} Thailand

\section{Article Info}

Article history:

Received Nov 15, 2020

Revised Jul 8, 2021

Accepted Aug 12, 2021

\section{Keywords:}

Change detection

Landsat

Machine learning

Toolbox

Water body classification

\begin{abstract}
Water bodies especially rivers are vital to existence of all lifeforms on Earth. Therefore, monitoring river areas and water bodies is essential. In the past, the monitoring relied essentially on manpower in surveying individual areas. However, there are limitations associated wih such surveys, e.g., tremendous amount of time and labour involved in expeditions. Presently, there have been accelerated development in remote sensing (RS) and artificial intelligence (AI) technology, particularly for change monitoring and detection in different areas globally. This research presents technical development of a toolbox for rivers classification and their change detection from Landsat images, by using water index analysis and four machine learning algorithms, which are KMeans, ISODATA, maximum likelihood classification (MLC), and support vector machine (SVM). Experimental findings indicated that all presented techniques were effective in detecting hydrological changes. The most accurate algorithm, nevertheless, for river classification was the SVM, with accuracy of $96.89 \%$, precision of $98.61 \%$, recall of $96.59 \%$, and F-measure of $97.59 \%$. Herein, it was demonstrated, in addition, that the developed toolbox was versatile and could be applied in rapid river change detection in other areas.
\end{abstract}

This is an open access article under the CC BY-SA license.

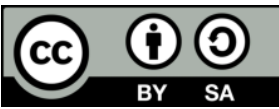

\section{Corresponding Author:}

Supattra Puttinaovarat

Faculty of Science and Industrial Technology

Prince of Songkla University

Surat Thani Campus, Surat Thani, Thailand, 84000

Email: supattra.p@psu.ac.th

\section{INTRODUCTION}

Information on water bodies is essential for both geographical and remote sensing applications in several aspects. For examples, it can be used for city planning and administration in specific sites [1], coastal erosion detection [2], water encroachment detection [3], river change detection [4], and flood analysis [5]. In the past, acquisition of water bodies or rivers data required human resources for site surveys, inevitably causing great delay and waste of budget, and taking significantly long time for data collection and processing. However, with continuous advances in remote sensing and computing technologies, processing of these hydrological data and relevant management processes can be executed ever much quickly, with virtually no need for human intervention. According to recent literature on remotely sensed data acquisition and processing, in particular, those based on image analysis, it was established that Landsat images were the 
most preferred modality in handling river data [6]-[10]. This is mainly because their data services are provided with no charge to users. Besides, Landsat images contain sufficient information for characterizing water bodies and particularly main rivers, for classification purposes, at reasonably high accuracy [6]-[10].

It is undeniable that water bodies or river shapes have underwent continuing changes, due to several geographic and meteorological causes, e.g., river erosion, caused by global climate change; natural disasters; higher water levels; subsidence; improper land uses, e.g., groundwater suction, and mountain forest invasion. These hydrological deteriorations have so far contributed to global and regional impacts in various aspects, at both economic and community levels [11]. They include loss of land value, occupational income, money for repairing and protection of riverbank houses and their reconstructions. Moreover, they also have adversarial impacts socially, such as resettlement and changing community ways of life, and environmentally, such as loss of soil resource, mangrove degradation, aquatic animal reduction, loss of riverbank landscapes, and tremendous expeditions on relocating people back to safety, construction remodeling, and administrating water prevention projects. Unfortunately, there is no exception for Surat Thani Province, where the Tapee River and others are present and hence under serious threat of river erosion. Therefore, the developments of effective tools for river change detection are important for preparing ourselves for these imminent transformations.

According to related studies, change detection is most frequently found employed in the context of land use and cover (LULC) [12]-[16], and coastline change analysis and detection [17]-[21]. Particularly, on detecting river change, remotely sensed (RS) satellite images and their processing were frequently employed [4], [22]. Thanks to its economic advantages, Landsat is one of the most frequently opted modalities. The images can be acquired free of charge from the USGS website. In addition, with high spectral resolution, main rivers can be characterized at great accuracy [6]-[10]. Nonetheless, for sub-river classification, which required greater fidelty, Landsat images are usually considered jointly with other RS data [23]-[26]. Based on imaging data, the common foundation of classification techniques is characterizing picture elements by their reflectance indices, and the most prevailing of which include NDWI, MNDWI, and AWEI. These indices are then classified by using state-of-the-art machine learning (ML), for instances, MLC and SVM [27]-[29]. In addition to research works, on processing these data for detecting river changes, ready to use software packages are also available. They are, for instances, ERDAS, and ArcGIS Desktop [30]-[31]. Thus far, an impeding factor, in exploiting these packages, is having to use step-by-step commands. They are not only taking long scripting time, but sometimes incompatible in partially supported functions. That being said, modern software developments have enabled automate scripts to be integrated in a form of toolboxes. For instance, ArcGIS desktop contains standard toolboxes pre-installed. Those specially designed and sophisticate functions can also be developed and integrated by using Model Builder. As such, the feature eliminates the need, for example, for manually inputting separate equations and control statements in a processing module. Nonetheless, to the best of our knowledge, there has no toolbox ever been developed for river classification and change detection during specific period. Therefore, this paper presents a development of such toolbox, based on differential indices, i.e., NDWI and MNDWI. River classification and detection were implemented by various ML techniques, i.e., ISODATA, K-means, MLC, and SVM.

The study area considered here was Surat Thai Province, located in the southern part of Thailand, with several main rivers flowing through, particularly Tapee River, Pumduang River, Kirirath River, and the Phunphin canal as shown in Figure 1. Currently, the area is subject to severe river erosion. The conditions hence call for an effective tool for assessing the topographic situations.

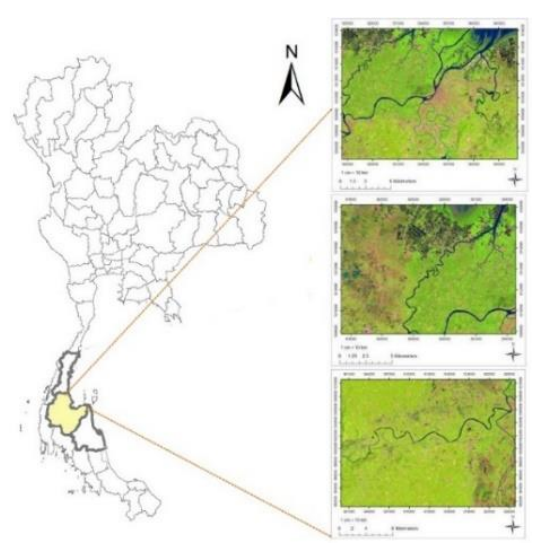

Figure 1. The study area is Surat Thani located in the south of Thailand (left) and some main rivers (right) 


\section{RESEARCH METHOD}

The main contribution of this paper is the development of an effective toolbox for river classification and its change detection. The toolbox consisted of six modular steps, i.e., data collection and preparation, water index calcuation, ML based river classification, accuracy assessment, and river change detection, and report presentation as shown in Figure 2. Each step is described in more detail the following sub-sections. River change detection algorithm in this paper shown in Table 1.

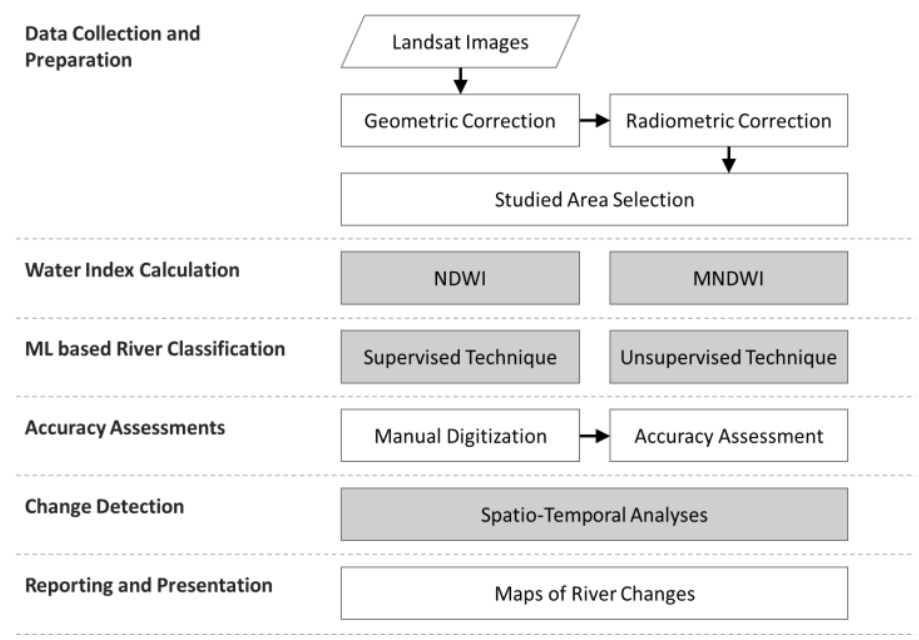

Figure 2. Diagram of the proposed methodology and the developed toolbox. It consists of data preparation, water index calculation, ML based river classification, accuracy assessment, change detection, and data reporting. Those highlighted in grey indicate the novelties of this study

Table 1. River change detection algorithm

\begin{tabular}{l}
\hline River Change Detection Algorithm \\
\hline Step 1: Input: Landat Satellite Image Dataset \\
Step 2: Geometric Correction \& Radiometric Correction \\
Step 3: Geoprocessing Analysis \\
Step 4: Water Index Calculation \\
NDWI = (NIR-MIR)/(NIR+MIR) \\
MNDWI = (Green-MIR)/(Green+MIR) \\
Step 5: Machine Learning Classification \\
IF ML is "Supervised" \\
Select Supervised Algorithm Name \\
Image Classify by Selected Algorithm \\
Return Image Classified Result \\
Select Unsupervised Algorithm Name \\
Image Classify by Selected Algorithm \\
Return Image Classified Result \\
Input: Images Clssified Rusult \\
River Chage Detection using Overlay Analysis \\
Ruturn Map of River changes
\end{tabular}

\subsection{Data collection and preparation}

Imaging data analyzed in this research were Landsat 8 and Sentinel-2 images, obtained from USGS website. Both radiometric and geometric errors in original images were first fixed by image correction. Then they were fed into Geoprocessing module, by which the locations and extents of studied areas were specified. Prior to processing these images by the developed toolbox, the ground truths of the rivers were prepared for accuracy evaluation and assessment. To this end, the corresponding LULC data were received from the Land Development Department of Thailand. 


\subsection{Water index calculation}

Subsequently, water features were required for training and evaluating ML classification modules. Following previous studies, it was accepted that two of the most suitable water indices for river extraction were NDWI and MNDWI. Empirical results reported in the linterature have demonstrated accurte and successful usecases of the indices. In this study, they were calculated from Landsat 8 images, using a toolbox in ArcGIS Desktop. Essentially, this step was considered preprocessing prior to moving onto the next important steps. The expressions and references of theses indices are summarized in Table 2.

Table 2. Water indices considered in this study

\begin{tabular}{|c|c|c|}
\hline Indices & Expressions & Description \\
\hline NDWI & NDWI $=($ NIR-MIR $) /($ NIR+MIR $)$ & NIR is a near infrared band \\
\hline MNDWI & MNDWI $=($ Green-MIR $) /($ Green+MIR $)$ & $\begin{array}{l}\text { MIR is a middle infrared band } \\
\text { Green is the green band }\end{array}$ \\
\hline
\end{tabular}

\subsection{River classification using machine learning}

Classification based on machine learning (ML) have widely been adopted in several similar studies. They may be categorized into unsupervised and supervised strategies. To determine the appropriate strategies for the present context, both categories were, therefore, benchmarked. For unsupervised classification, KMeans and ISODATA were used, while for supervised classification, MLC and SVM were considered. During 2 class's classification, each pixel in an image would be labelled either of river or non-river, depending on its water indices. While in the first category, no training data was needed, 1,000 points per classes were prepared for the second one, to create the respective supervised ML models. The detailed description and properties of each ML algorithm are described as follow.

\subsubsection{K-Means}

This algorithm is one of the unsupervised learning. With K-Means, objects are parted into $K$ clusters, each of which is represented by its mean, or centroid. It is used to measure the distance between data within the same cluster. As the first step, the number of expected clusters $(\mathrm{K})$ and their associated means must be initialized. Next, data clusters and their relationship are built, among those data points closest to the respective centroids. Subsequently, a centroid is recalculated, for each cluster, based on the newly updated membership. If a centroid of any cluster is relocated as a result, its membership is also reconsidered, based on nearest data points. These reciprocal updates are itearated, until no change is made on any of the $K$ centroids. In the proposed model, K-Means included parameters as reveal in Table 3.

Table 3. K-Means parameter settings

\begin{tabular}{ll}
\hline Parameter & Value \\
\hline numClusters & 2 \\
distanceFunction & Euclidean \\
Iteration & 500 \\
\hline
\end{tabular}

\subsubsection{ISODATA}

ISODATA is another unsupervised learning technique, mostly employed in applications involving satellite or aerial images. With this technique, wavelength distancing was considered along with several pixel classification repetitions, each of which consists of statistical re-calculation and re-classification. Once a user has specified the number of classes and their means, each pixel was assigned to a cluster based on its shortest distance. Unlike K-Means, the new centroids would then move toward the statistically rearranged data, so as to resolve K-Means limitation. This process is repeated until convergence is reached. In the proposed model, ISODATA included parameters as reveal in Table 4.

Table 4. ISODATA parameter settings

\begin{tabular}{ll}
\hline Parameter & Value \\
\hline numClusters & 2 \\
ChangeThreshold (\%) & 5 \\
Iteration & 500 \\
\hline
\end{tabular}




\subsubsection{MLC}

This ML is considered a supervised learning technique. It was widely used in processing of remote sensing data. With this technique, the probability of each data point being predicted as a class is determined by class mean and covariance. More specifically, given a probability distribution of all involving classes, decision is made for a given point being associated with any class, by comparing respective class probability in multi-class mixture models. Accordingly, in multi-spectral remotely sensed images, each pixel is classified jointly by the probability in specific frequency domains. In the proposed model, MLC included parameters as reveal in Table 5.

\begin{tabular}{ll} 
Table 5. MLC parameter settings \\
\hline Parameter & Value \\
\hline numClassses & 2 \\
reject fraction & 0.01 \\
Iteration & 500 \\
\hline
\end{tabular}

\subsubsection{SVM}

This supervised ML is specially designed for building a highly generalized classifier. Support vector machine (SVM) performs best with unseen dataset, both with low and high dimensionality. In the former case, adjustment was made to the classifier on the input space by trivial discriminant, while in the other case, it was made by more flexible adjustment, called kernel function, on the reducible feature space. Either case, the resultant is expressed as a set of optimal hyperplanes that jointly separate samples in different classes. Therefore, SVM is transparent and highly scalable, i.e., with respect to the complexity of the input data. In addition, unlike other MLs, data need not to be normally distributed, nor do they need to be sampled by large amount. Compared to MLs with similar computational complexity, SVM is reported to be much efficient and accurate, in many applications. In the proposed model, SVM included parameters as reveal in Table 6.

Table 6. SVM parameter settings

\begin{tabular}{ll}
\hline Parameter & Value \\
\hline numClassses & 2 \\
Kernel & Radial Basis Function (RBF) \\
Iteration & 500 \\
\hline
\end{tabular}

\subsection{Accuracy assessments}

In this paper, the accuracy of the river classification by the abovementioned ML techniques were assessed by comparing the resultant extractions against the manually digitized LULC data, provided by the Land Development Department of Thailand. To this end, four evaluation metrices, i.e., Accuracy, Recall, Precision and F-measure, were calculated. Then, the best performing with the highest measures was chosen for the river classification toolbox. The extracted rivers, at each annual period, would then be forwarded to the change detection module, described in the next sub-section. All other relevant accuracy assessment metrics are revealed in Table 7 .

Table 7. Accuracy assessment expressions

\begin{tabular}{lll}
\hline Evaluation method & \multicolumn{1}{c}{ Expression } & Description \\
\hline Accuracy & $\mathrm{CCI}=(\mathrm{TP}+\mathrm{TN}) /(\mathrm{TP}+\mathrm{TN}+\mathrm{FP}+\mathrm{FN})$ & TP is True Positive \\
Precision & Precision $=\mathrm{TP} /(\mathrm{TP}+\mathrm{FP})$ & TN is True Negative \\
Recall & Recall $=\mathrm{TP} /(\mathrm{TP}+\mathrm{FN})$ & FP is False Positive \\
F-measure & F-measure $=(2 \times$ Precision $\times$ Recall $) /($ Precision+Recall $)$ & FN is False Negative \\
\hline
\end{tabular}

\subsection{River change detection}

On detecting river changes, the developed module was validated on Landsat imaging data acquired at the years 1999, 2006, 2011, and 2017. The changes between subsequent annual periods was determined by comparing extracted rivers at the respective years. This comparison was made using raster operations on their differences and then implemented in a toolbox. These changed areas, i.e., transitions, were characterized and reported on a map, as either from river areas to non-river ones, or vice versa. 


\section{RESULTS AND DISCUSSION}

The experimental results reported in this paper are divided into three main parts, which are i) the toolbox development for river classification and change detection, ii) the river classification, and iii) the river change detection. For the first part, the toolbox was developed by using the model builder. Its screen shots are shown in Figure 3. The toolbox consisted of three functions, i.e., water index calculation, river classification, and river change detection. In the first function, water indices consisted of NDWI and MNDWI calculations. Likewise, river classification consisted of different ML algorithms, i.e., MLC, ISODATA, SVM, and K-Means. Finally, the change detection simply exploited built-in functionalities. Apparently, this toolbox could be extended and applied to other areas. By not operating directly on regular ArcGIS Desktop, a user is not required perform these functions by clicking and typing step-by-step commands, from the start to the end. Last but not least, using toolbox was straightforward and its data processing on studied areas was very fast.
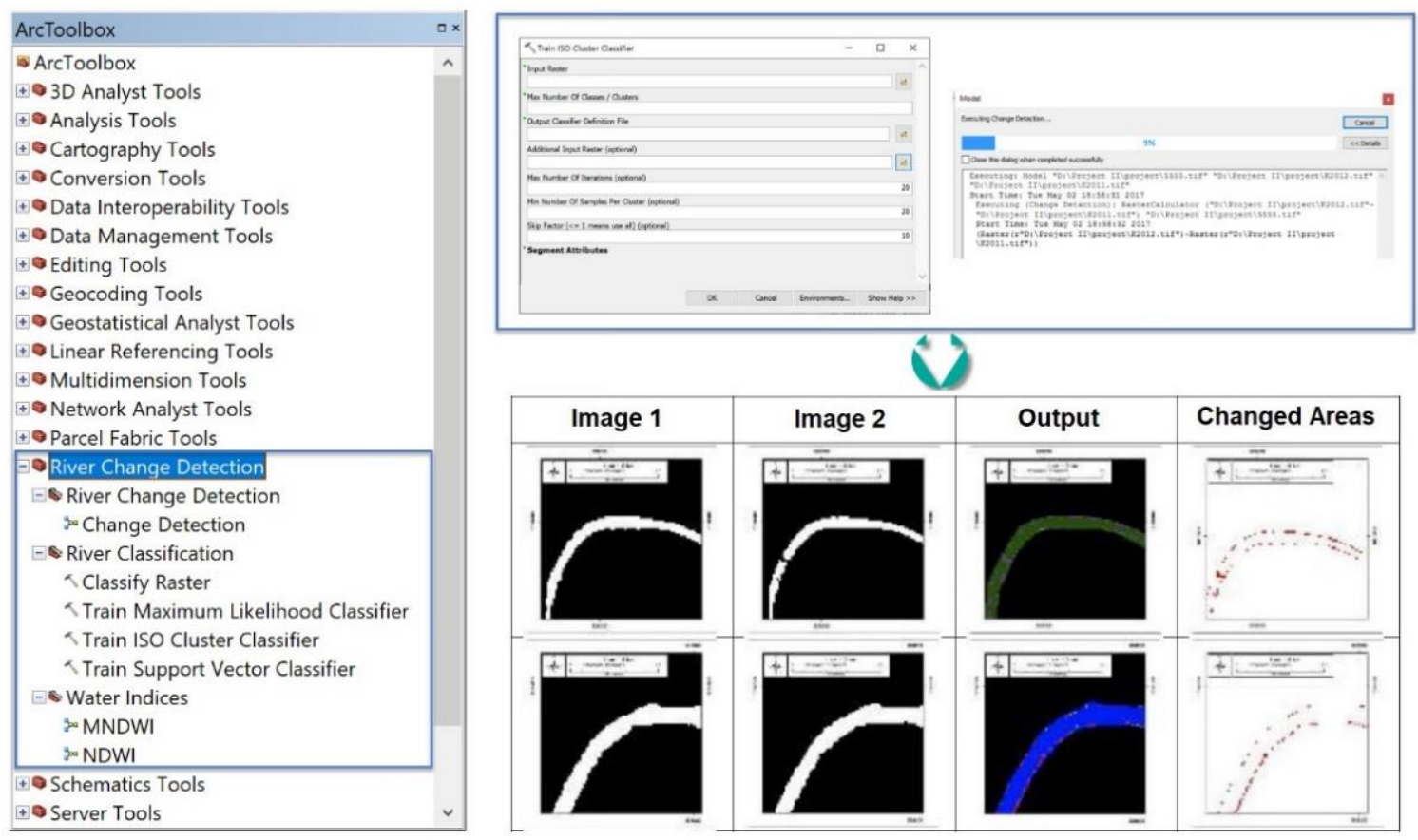

Figure 3. Screen shots of the developed toolbox for river classification and its change detection

The results of the second part, i.e., river classification based on water index are reported in Figure 4. Therein, classification of different ML techniques, both supervised and unsupervised, are compared at four different annual periods. By visual assessment, it is evidient that MNDWI yielded more accurate results than NDWI, both by supervised and unsupervised technqiues. Therefore, MNDWI was chosen and its metrices were calculated, for each technique, and presented in Table 8 and Figure 5. According to these objective evaluations, it was found that the best performing algorithm in all four values (Accuracy, Recall, Precision and F-measure) was SVM, with accuracy $=96.89 \%$, precision $=98.61 \%$, recall $=96.59 \%$, and F-measure $=$ $97.59 \%$. It was followed by MLC, ISODATA, and K-means, respectively. Therefore, this research employed MNDWI and SVM algorithms for river classification, and developed the river change detection toolbox, accordingly. 


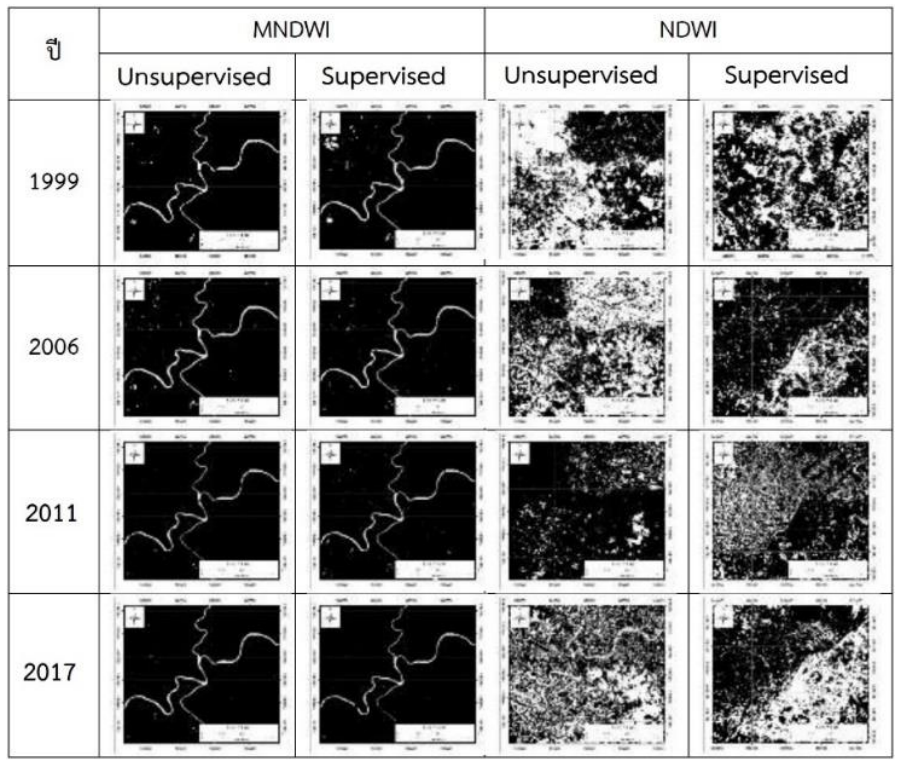

Figure 4. Unsupervised and supervised classification results based on MNDWI and NDWI indices.

Table 8. Accuracy assessment

\begin{tabular}{lllll}
\hline Algorithm & Accuracy & Precision & Recall & F-measure \\
\hline ISODATA & 94.56 & 97.88 & 95.29 & 96.57 \\
K-means & 93.89 & 96.66 & 95.60 & 96.22 \\
MLC & 95.12 & 98.35 & 94.55 & 96.49 \\
SVM & 96.89 & 98.61 & 96.59 & 97.59 \\
\hline
\end{tabular}
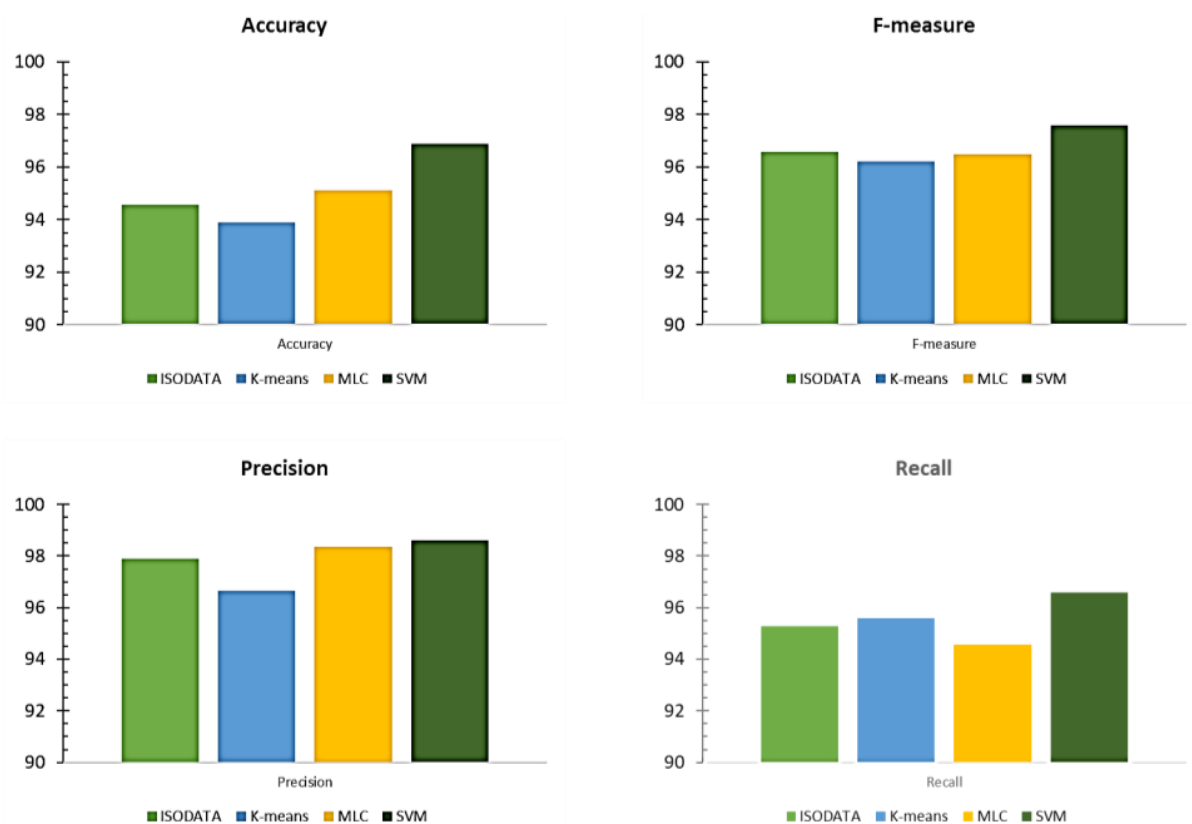

Figure 5. Comparisons of accuracy assessment

Finally, the river change detection results are reported for the study areas, surrounding three major rivers and canals in Surat Thani Province. They are Tapee River, Pumduang River, and Phunphin Canal. Their detected changes are displayed in Figures 6-8. These augmented satellite images were overlaid by 
water bodies extracted at the years 1999, 2006, 2011, and 2017 AD, respectively. It can be clearly noticed from the figures that river changes in all considered areas took similar pattern. Specifically, there existed progressive erosion in all rivers and canal as they got narrower. Particularly, this was much pronounced for Phunphin canal than other areas. In summary, the developed change detection toolbox of rivers by processing their satellite images was found beneficial in various aspects. Not only that it was easy to use and getting used to, but the toolbox could also reduce manpower and time required for actual onsite surveys. As such, it could offer government agencies, sufficient information for, and an efficient means of planning, following up, and monitoring changes, so that they can prepare for upcoming geographical changes. Data obtained from change analysis are useful for predicting the likelihood of other relevant change, such as flooding and landslides. Compared to the previous river classification and change detection [22], it was found that this research has the strength to investigate changes in river regions without using step-by-step commands in ArcGIS. It also reduces the processing time.

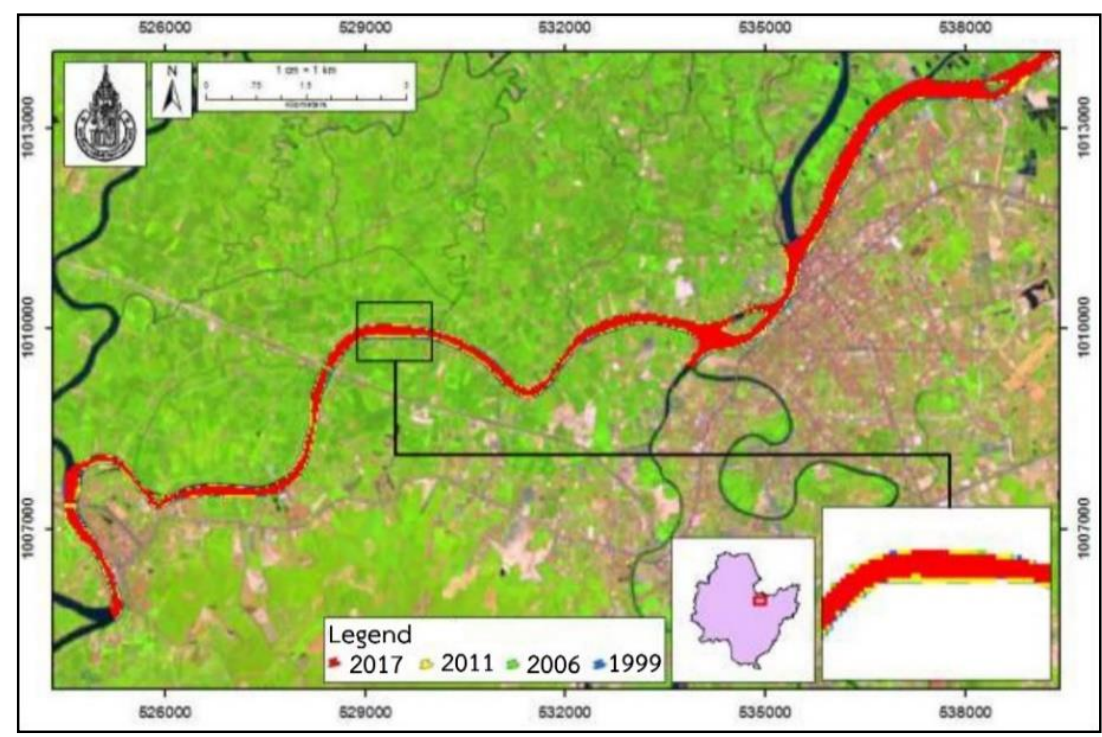

Figure 6. Augmented satellite image of Tapee River change detection

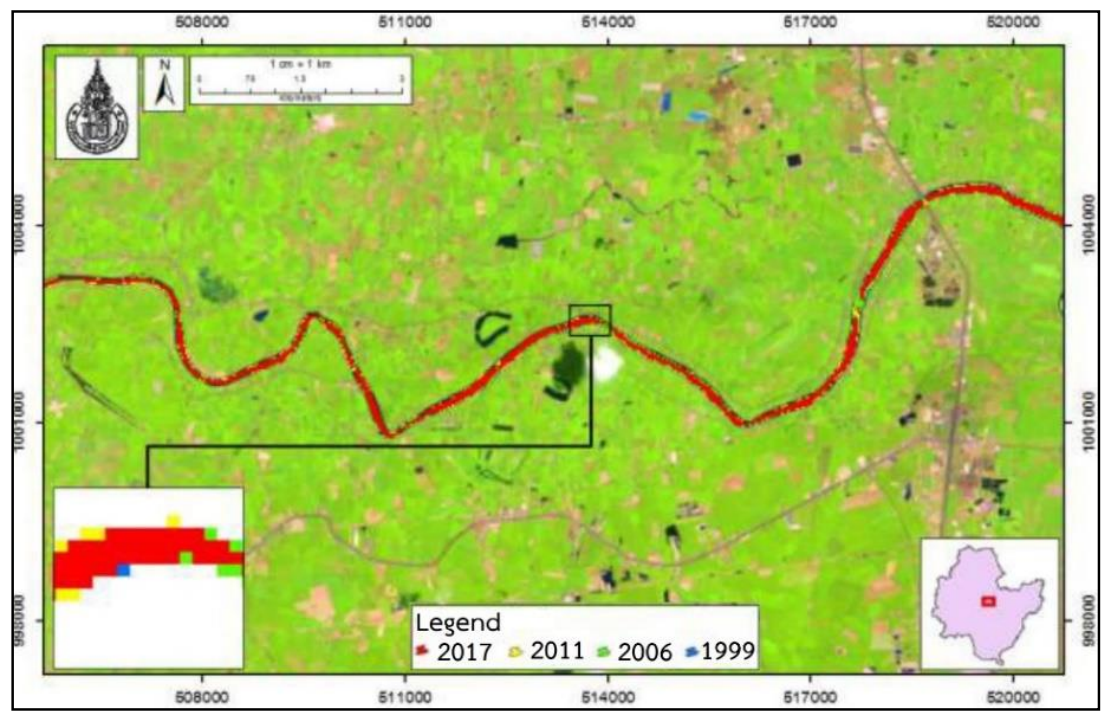

Figure 7. Augmented satellite image of Pumduang River change detection 


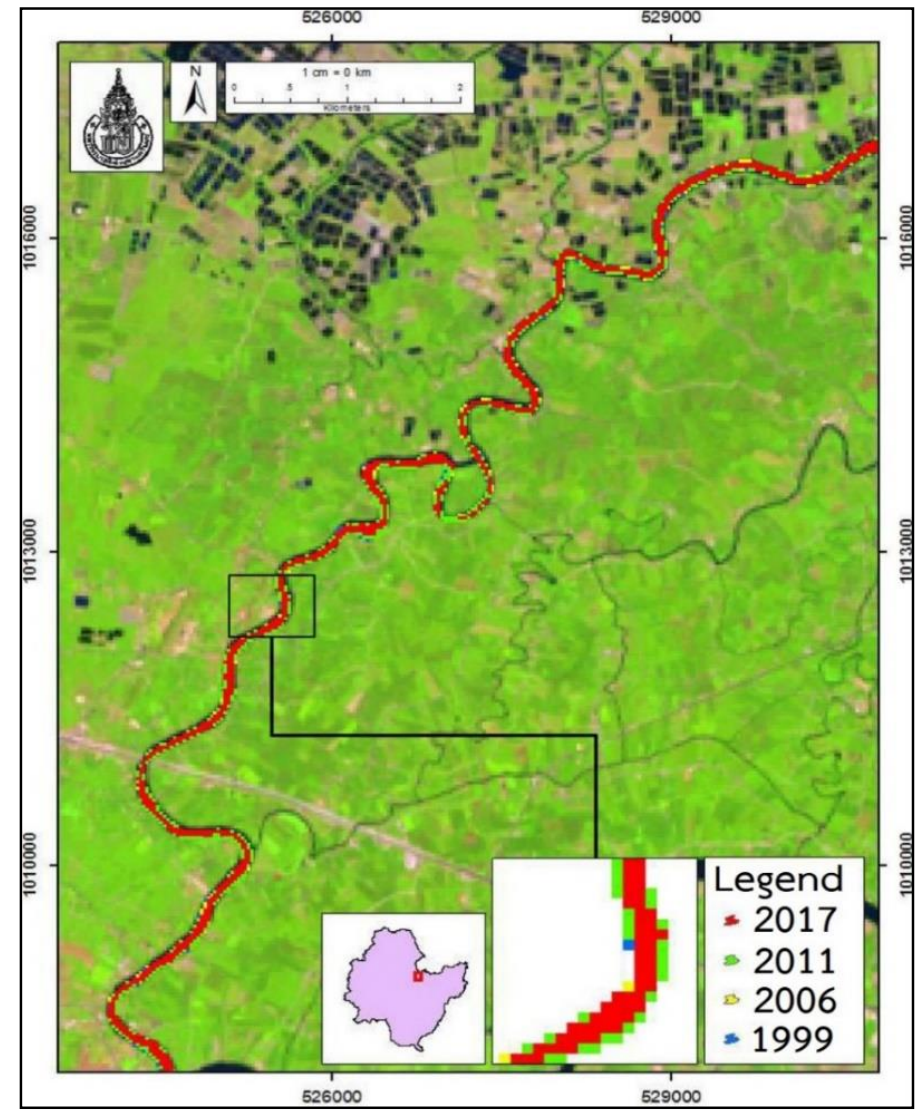

Figure 8. Augmented satellite image of Phunphin canal change detection

\section{CONCLUSION}

This research presented river change detection using Landsat image processing, and development of a toolbox for that purpose. Both supervised and unsupervised classification ML were applied to water index, calculated from Landsat 8 . The experimental results showed that river classification could be made with high accuracy. Based on the studied areas, the best performing algorithm was SVM, with accuracy $96.89 \%$, followed by MLC, and others. River changes could also be detected in each particular period, from years 1999-2017 AD. It was evident in all considered rivers and cannel that they got narrow than in the past, due to riverbank having undergone erosion. Thanks to the developed toolbox, no manual intervention was required by a user, at any step in the whole process. It is also worth noted that, thanks to versatility of the ArcGIS platform, the toolbox can be extended and applied to other areas, subject to data availability. Since no other assumptions were imposed on the toolbox development, satellite images of higher resolution, for example, can be employed to gain higher classification resolution. The change detection proposed herein by comparing extracted water bodies of aligned data at different periods is straightforward and efficient. It can greatly reduce costs of onsite surveys. Thus far, the current study remained limited by only using data from NIR band. Future directions include predicting the likelihood of other related hydrological changes.

\section{ACKNOWLEDGEMENTS}

The authors are deeply grateful to the Faculty of Science and Industrial Technology, Prince of Songkla University, Surat Thani campus, Thailand. This research was financially supported by Prince of Songkla University, Surat Thani Campus.

\section{REFERENCES}

[1] A. Lehner and T. Blaschke, "A generic classification scheme for urban structure types," Remote Sensing, vol. 11, no. 2, 2019, Art. No. 173, doi: 10.3390/rs11020173. 
[2] T. A. Łabuz, "Environmental impacts-coastal erosion and coastline changes," In Second Assessment of Climate Change for the Baltic Sea Basin, pp. 381-396, 2015, doi: 10.1007/978-3-319-16006-1_20.

[3] E. Honlah, A. Yao Segbefia, D. Odame Appiah, M. Mensah and P. O. Atakora, "Effects of water hyacinth invasion on the health of the communities, and the education of children along River Tano and Abby-Tano Lagoon in Ghana," Cogent Social Sciences, vol. 5, no. 1, Art. No. 1619652, 2019, doi: 10.1080/23311886.2019.1619652.

[4] W. Xin, T. Can, W. Wei and L. Ji, "Change Detection of Water Resources via Remote Sensing: An LV-NSCT Approach," Applied Sciences, vol. 9, no. 6, 2019, Art. No. 1223, doi: 10.3390/app9061223.

[5] S. Puttinaovarat and P. Horkaew, "Flood Forecasting system based on integrated big and crowdsource data by using machine learning techniques, "IEEE Access, vol. 8, pp. 5885-5905, 2020, doi: 10.1109/access.2019.2963819.

[6] B. C. Ko, H. H. Kim and J. Y. Nam, "Classification of potential water bodies using Landsat 8 OLI and a combination of two boosted random forest classifiers," Sensors, vol. 15, no. 6, pp. 13763-13777, 2015, doi: 10.3390/s150613763.

[7] K. Mishra and P. Prasad, "Automatic extraction of water bodies from Landsat imagery using perceptron model," Journal of Computational Environmental Sciences, vol. 2015, 2015, doi: 10.1155/2015/903465.

[8] J. P. Mondejar and A. F. Tongco, "Near infrared band of Landsat 8 as water index: A case study around Cordova and Lapu-Lapu City, Cebu, Philippines," Sustainable Environment Research, vol. 29, no. 1, 2019, Art. No. 16, doi: 10.1186/s42834-019-0016-5.

[9] S. Puttinaovarat and P. Horkaew, "Internetworking flood disaster mitigation system based on remote sensing and mobile GIS," Geomatics, Natural Hazards and Risk, vol. 11, no. 1, pp. 1886-1911, 2020, doi: 10.1080/19475705.2020.1815869.

[10] F. Pan, X. Xi and C. Wang, "A comparative study of water indices and image classification algorithms for mapping inland surface water bodies using landsat imagery," Remote Sensing, vol. 12, no. 10, 2020, Art. No. 1611, doi: 10.20944/preprints201911.0218.v1.

[11] M. A. H. Bhuiyan, S. D. U. Islam and G. Azam, "Exploring impacts and livelihood vulnerability of riverbank erosion hazard among rural household along the river Padma of Bangladesh," Environmental Systems Research, vol. 6, no. 1, 25, 2017, doi: 10.1186/s40068-017-0102-9.

[12] J. F. Mas, R. Lemoine-Rodríguez, R. González-López, J. López-Sánchez, A. Piña-Garduño and E. Herrera-Flores, "Land use/land cover change detection combining automatic processing and visual interpretation," European Journal of Remote Sensing, vol. 50, no. 1, pp. 626-635, 2017, doi: 10.1080/22797254.2017.1387505.

[13] J. S. Alawamy, S. K. Balasundram, A. H. M. Hanif and C. T. B. Sung, "Detecting and analyzing land use and land cover changes in the region of Al-Jabal Al-Akhdar, Libya Using Time-series Landsat Data from 1985 to 2017," Sustainability, vol. 12, no. 11, 2020, Art. No. 4490, doi: 10.3390/su12114490.

[14] J. Spruce, J. Bolten, I. N. Mohammed, R. Srinivasan and V. Lakshmi, "Mapping land use land cover change in the lower mekong basin from 1997 to 2010," Frontiers in environmental science, vol. 8, 2020, Art. No. 21, doi: $10.3389 /$ fenvs.2020.00021.

[15] S. R. Aldhshan and H. Z. M. Shafri, "Change detection on land use/land cover and land surface temperature using spatiotemporal data of Landsat: a case study of Gaza Strip," Arabian Journal of Geosciences, vol. 12, no. 14, 2019, Art. No. 443, doi: 10.1007/s12517-019-4597-4.

[16] W. Chen, Y. Wang, X. Li, Y. Zou, Y. Liao and J. Yang, "Land use/land cover change and driving effects of water environment system in Dunhuang Basin, northwestern China," Environmental earth sciences, vol. 75, no. 12, 1027, 2016, doi: 10.1007/s12665-016-5809-9.

[17] K. Topouzelis, A. Papakonstantinou and M. Doukari, "Coastline change detection using Unmanned Aerial Vehicles and image processing technique," Fresen. Environ. Bull. vol. 26, pp. 5564-5571, 2017, doi: 10.1109/siu49456.2020.9302449.

[18] X. Wang, Y. Liu, F. Ling, Y. Liu and F. Fang, "Spatio-temporal change detection of Ningbo coastline using Landsat time-series images during 1976-2015," ISPRS International Journal of Geo-Information, vol. 6, no. 3, 2017, Art. No. 68, doi: 10.3390/ijgi6030068

[19] M. K. Ghosh, L. Kumar and C. Roy, "Monitoring the coastline change of Hatiya Island in Bangladesh using remote sensing techniques," ISPRS Journal of Photogrammetry and Remote Sensing, vol. 101, pp. 137-144, 2015, doi: 10.1016/j.isprsjprs.2014.12.009.

[20] A. G. Shaima and O. Hegazy, "Coastline change detection on tehama city using remote sensing and cloud computing," International Journal of Computer Science and Information Technology Research, vol. 2, no. 3, pp. 499-505, 2014, doi: 10.1016/j.isprsjprs.2014.12.009.

[21] M. K. Kim, H. G. Sohn, S. P. Kim and H. S. Jang, "Automatic coastline extraction and change detection monitoring using Landsat imagery," Journal of Korean Society for Geospatial Information System, vol. 21, no. 4, pp. 45-53, 2013, doi: 10.7319/kogsis.2013.21.4.045.

[22] S. P. Aher, S. I. Bairagi, P. P. Deshmukh and R. D. Gaikwad, "River change detection and bank erosion identification using topographical and remote sensing data," International Journal of Applied Information Systems, vol. 2, pp. 1-7, 2012.

[23] P. Horkaew and S. Puttinaovarat, "Entropy-Based Fusion of Water Indices and DSM Derivatives for Automatic Water Surfaces Extraction and Flood Monitoring," ISPRS International Journal of Geo-Information, vol. 6, no. 10, Art. No. 301, 2017, doi: 10.3390/ijgi6100301.

[24] F. Ahmad, L. Goparaju and A. Qayum, "Natural resource mapping using landsat and lidar towards identifying digital elevation, digital surface and canopy height models," Int J Environ Sci Nat Res, vol. 2, 2017, Art. No. 555580, doi: 10.19080/ijesnr.2017.02.555580. 
[25] B. C. Naik, and B. Anuradha, "Extraction of Water-body Area from High-resolution Landsat Imagery," International Journal of Electrical and Computer Engineering (IJECE), vol. 8, no. 6, pp. 4111-4119, 2018, doi: 10.11591/ijece.v8i6.pp4111-4119.

[26] S. L. Kumar Reddy et al., "An index based road feature extraction from LANDSAT-8 OLI images," International Journal of Electrical and Computer Engineering (IJECE), Vol. 11, no. 2, pp. 1319-1336, 2021, doi: 10.11591/ijece.v11i2.pp1319-1336

[27] E. Guermazi, M. Bouaziz and M. Zairi, "Water irrigation management using remote sensing techniques: a case study in Central Tunisia," Environmental Earth Sciences, vol. 75, no. 3, 202, 2016, doi: 10.1007/s12665-015-4804-x.

[28] N. Yagmur, N. Musaoglu and G. Taskin, "Detection of shallow water area with machine learning algorithms," Int. Archives of the Photogrammetry, Remote Sensing and Spatial Information Sciences, pp. 1269-1273, 2019.

[29] J. J. H. Harikiran, "Hyperspectral image classification using support vector machines," IAES International Journal of Artificial Intelligence (IJAI), vol. 9, no. 4, pp. 684-690, 2020, doi: 10.11591/ijai.v9.i4.pp684-690.

[30] M. Agaton, Y. Setiawan and H. Effendi,"Land use/land cover change detection in an urban watershed: a case study of upper Citarum Watershed, West Java Province, Indonesia," Procedia Environmental Sciences, vol. 33, pp. 654-660, 2016, doi: 10.1016/j.proenv.2016.03.120.

[31] Bajjali, William, ArcGIS for environmental and water issues, Springer, 2017.

\section{BIOGRAPHIES OF AUTHORS}

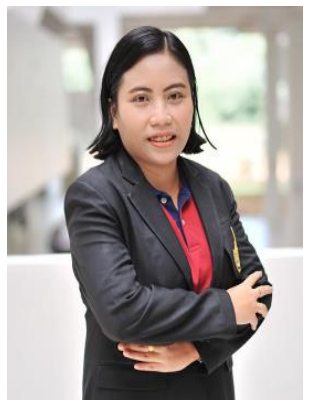

Supattra Puttinaovarat is an assistant professor at the Faculty of Science and Industrial Technology, Prince of Songkla University, Surat Thani Campus. Her research interest includes Geographic Information System, Remote Sensing, Machine Learning, and Information Technology.

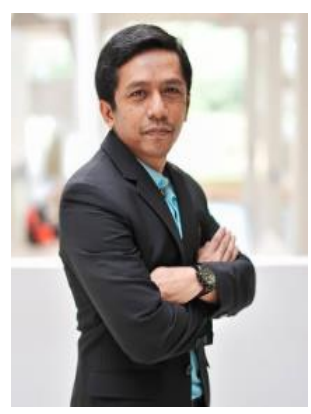

Aekarat Saeliw is a lecturer at Faculty of Science and Industrial Technology, Prince of Songkla University, Surat Thani Campus. His research interest includes Information Technology and Mobile Application.

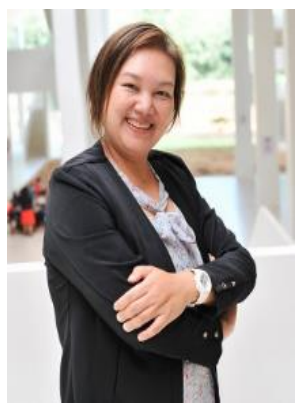

Siwipa Pruitikanee is a lecturer at the Faculty of Science and Industrial Technology, Prince of Songkla University, Surat Thani Campus. Her research interest includes Information Technology and Mobile Application. 


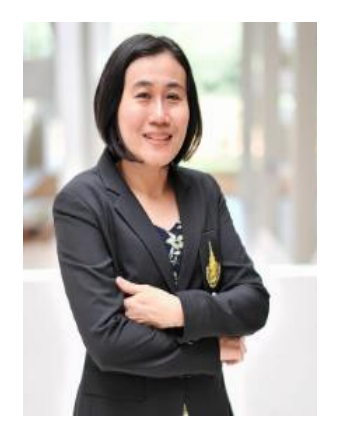

Jinda Kongcharoen is an assistant professor at the Faculty of Science and Industrial Technology, Prince of Songkla University, Surat Thani Campus. Her research interest includes Statistics, Applied Statistics, and Information Technology.

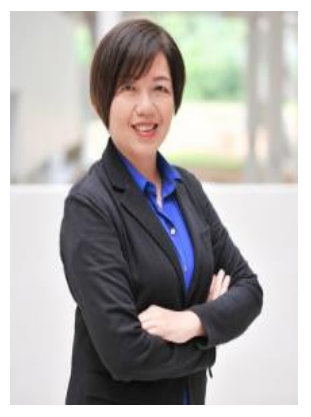

Supaporn Chai-Arayalert is a lecturer at the Faculty of Science and Industrial Technology at Prince of Songkla University, Thailand. Her current research interests include knowledge management, Green IT, IT project management, e-commerce, and business information systems.

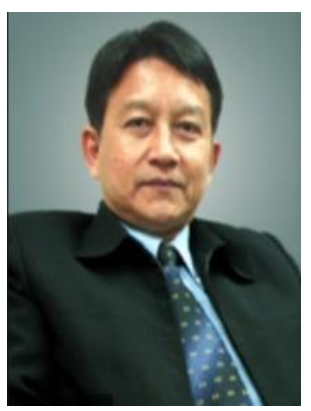

Kanit Khaimook is an associate professor at Ramkhamhaeng University, Bangkok, Thailand. His research interest includes Information Technology and Statistics.

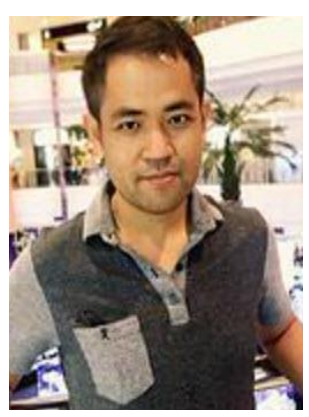

Paramate Horkaew is an assistant professor at the School of Computer Engineering, Suranaree University of Technology, Thailand. His main research interests include Digital Geometry Processing with applications in Remote Sensing and Geographical Information Systems, Computer Vision, and Graphics. 